\title{
Boron Neutron Capture Therapy (BNCT) for Treatment of Multiple Tumor Models
}

\section{Aslam A Khan*}

Department of Biochemistry, University of Missouri, Columbia, USA

\section{Introduction}

Radiation therapy is the therapeutic modality in which high energy radiations are used to kill tumor cells [1]. Conventional radiation therapy is a whole body radiation process resulting in numerous side effects, highlighting the need to identify alternative targeted therapy [1]. BNCT is a targeted therapy in which cancer cells accumulate boron and are subsequently bombarded with neutrons [2]. During this process, boron accepts the neutron and breaks into two nuclei, Lithium and Helium, releasing energy in the process. The path traveled by the released energy particles is approximately $10 \mu \mathrm{m}$ in diameter, which is approximately the size of a single cancer cell [3]. The preferential boron accumulation by cancer cells and the short range of energy particles make BNCT a far more targeted therapy than whole body irradiation [3]. As a consequence of its relative specificity, BNCT produces fewer side effects than traditional radiation therapy [4]. One important aspect in the advancement of BNCT is the development of compounds which can deliver a high boron payload to the tumors. Accordingly, Dr. Hawthorne developed compounds Na3[1-(2'B10H9)-2-NH3B10H8] (MAC) and K[nido-7-CH3(CH2)15-7,8C2B9H11] (TAC) that can now deliver high concentration of boron into tumor cells. While other boron compounds had been developed previously, they all suffered from a lack of tumor targetability [5]. To circumvent this decreased targetability, compounds developed by Dr. Hawthorne were engineered such that hydrophobic compound is incorporated into the lipid membrane while the hydrophilic compound is encapsulated within the core of the rationally designed liposomes.

Treatment of murine mammary tumor (EMT-6) usingliposomes incorporating TAC and MAC: Previous studies have utilized BNCT for the treatment of glioblastoma. We applied this same therapy for the targeted treatment of murine mammary tumor utilizing the liposome- encapsulated TAC and MAC boron compounds. Because the effectiveness of BNCT is directly proportional to the accumulation of boron in tumor tissue, the limiting factor for proceeding with the therapy was the biodistribution of boron within the tumor tissue. We observed a wide range of biodistribution patterns where some tumors demonstrated a very high boron payload while others had a negligible payload. To circumvent these biodistribution discrepancies, we grew EMT-6 tumors to controlled sizes, from $100 \mathrm{mg}-500 \mathrm{mg}$ and MACTAC liposomes were delivered via tail vein injection. We observed that tumor biodistribution was dependent on tumor size, where smaller tumors $(100 \mathrm{mg})$ accumulated higher boron payload and while larger tumors (500 mg) accumulated negligible boron payload (Unpublished data). Subsequently, we irradiated mice bearing EMT-6 tumors and observed a significant reduction in tumor sizes [6]. The mechanism of tumor reduction following irradiation was determined to be a combination of both apoptosis and necrosis of the tumor cells (Unpublished data). While we did observe the necessity of very high boron payloads to induce tumor reduction following irradiation, we successfully demonstrated the ability to inhibit tumor growth using liposome encapsulated boron compounds.

\section{Comparative Study of Mammary Tumor and Colon Tumor Using Liposome Incorporated TAC/MAC}

Following our studies using murine mammary tumors, we further investigated the use of boron compound liposomes in oral cancer using the hamster cheek pouch model where our results were essentially reproduced in a different tumor model. Next, we investigated the use of MAC-TAC liposomes in a colorectal cancer model that is resistant to standard chemotherapy. In these published studies, we compared the biodistribution of MAC-TAC liposomes in murine mammary tumors and colorectal (CT26) tumors and observed that the boron payload was lower in CT26 tumors, as compared to EMT-6. This lower boron payload could be due to differences of vascularization of these tumors. Irradiation of mice bearing either EMT- 6 or CT-26 tumors revealed that the tumor growth inhibition was significantly higher in CT26 tumors, as compared to EMT-6 tumors. BNCT has primarily been used for the treatment of glioblastomas; however, our studies demonstrate that BNCT can be a therapeutic modality for other tumor models and that MAC-TAC liposomes can deliver high boron payloads to tumor tissue.

\section{Possible Future Directions in BNCT}

I am of the opinion we need to reconsider the importance of BNCT which has been fading away quickly. It is important to continue to design smart boron compounds and delivery vehicles that can accumulate in, or deliver boron specifically to, tumor tissue. I have previously published a study with Dr. Hawthorne where carboplatin was appended to a boron closomers, which delivered a high drug payload into cancer cells [7]. We called these molecules drug delivery system (DDS). These drug delivery systems were able to accumulate in nuclei of the cancer cells, suggesting a close proximity of boron to DNA. As demonstrated in the literature, DNA damage is the basis of BNCT, however, there are currently no boron compounds that are able to directly target the nucleus. Perhaps this is why BNCT as a therapeutic modality has failed to deliver the desired results. Many cancers are treated with cisplatin-based radiation therapy, where patients are injected with cisplatin first followed by radiation therapy. DDS like the carboplatin appended closomers will hold more potential in future where the effect will be two-fold: carboplatin will either sensitize these tumors to radiation or act as chemotherapy agent and irradiation with neutrons will result in BNCT. This would represent a far more targeted therapy than traditional whole body radiation therapy.

*Corresponding author: Aslam A Khan, Department of Biochemistry, University of Missouri, University of Missouri, Columbia, USA, Tel: 573-8827823; E-mail: khanasl@missouri.edu

Received August 11, 2017; Accepted August 26, 2017; Published September 03, 2017

Citation: Khan AA (2017) Boron Neutron Capture Therapy (BNCT) for Treatment of Multiple Tumor Models. J Oncol Transl Res 3: 116. doi: 10.4172/24762261.1000116

Copyright: (c) 2017 Khan AA. This is an open-access article distributed under the terms of the Creative Commons Attribution License, which permits unrestricted use, distribution, and reproduction in any medium, provided the original author and source are credited. 
Citation: Khan AA (2017) Boron Neutron Capture Therapy (BNCT) for Treatment of Multiple Tumor Models. J Oncol Transl Res 3: 116. doi: 10.4172/2476-2261.1000116

Page 2 of 2

\section{References}

1. Berkey FJ (2010) Managing the adverse effects of radiation therapy. Am Fam Physician 82: 381-394.

2. Dorn RV (1994) Boron neutron capture therapy (BNCT): A radiation oncology perspective. Int J Radiat Oncol Biol Phys 28: 1189-1201.

3. Coderre JA (2003) Boron neutron capture therapy: Cellular targeting of high linear energy transfer radiation. Technol Cancer Res Treat 2: 355-375.

4. Slatkin DN (1991) A history of boron neutron capture therapy of brain tumours Postulation of a brain radiation dose tolerance limit. Brain 114: 1609-1629.
5. Ichikawa H, Taniguchi E, Fujimoto T, Fukumori Y (2009) Biodistribution of BPA and BSH after single, repeated and simultaneous administrations for neutroncapture therapy of cancer. Appl Radiat Isot 67: S111-114.

6. Kueffer PJ (2013) Boron neutron capture therapy demonstrated in mice bearing EMT6 tumors following selective delivery of boron by rationally designed liposomes. Proc Natl Acad Sci 110: 6512-6517.

7. Bondarev $\mathrm{O}$ (2013) Synthesis of [closo-B12(OH)11NH3]-: A new heterobifunctional dodecaborane scaffold for drug delivery applications. J Am Chem Soc 135: 13204-13211. 